\title{
The 4E-BP1/eIF4E ratio is a determinant for rapamycin response in esophageal cancer cells
}

\author{
Han-Shui Hsu, MD, PhD, , ,b Ming-Hsien Lin, MD, ${ }^{\text {,d }}$ Yi-Hua Jang, MS, ${ }^{\mathrm{e}}$ Ting-Ting Kuo, MS, \\ Chen-Chi Liu, MD, ${ }^{\mathrm{f}}$ and Tzu-Hao Cheng, $\mathrm{PhD}^{\mathrm{e}}$
}

Objectives: Rapamycin inhibits products of molecular pathways in esophageal squamous cell carcinoma and
limits tumor cell growth by targeting 4E-BP1- and eIF4E-dependent gene translation. In this study, we investi-
gate the influence of 4E-BP1-to-eIF4E ratio on rapamycin response in esophageal squamous cell carcinoma
cells, and the underlying mechanism is discussed.

\begin{abstract}
Methods: The response to rapamycin treatment was examined in 6 esophageal cancer cell lines. Adjustment of the 4E-BP1/eIF4E ratio was carried out by knockdown or overexpression of 4E-BP1 and eIF4E. The relationship between Egr-1 and 4E-BP1 expression in esophageal cancer cells was also studied.
\end{abstract}

\begin{abstract}
Results: The 4E-BP1/eIF4E ratio was adjusted to evaluate the response to rapamycin treatment in TE1 and TE2 esophageal cancer cells. TE2 cells are sensitized to rapamycin treatment after overexpression of 4E-BP1 or knockdown of eIF4E; TE1 cells become resistant to rapamycin after knockdown of 4E-BP1 or overexpression of eIF4E. These data suggest that the 4E-BP1/eIF4E ratio is a determinant for the response of TE1 and TE2 cells to rapamycin treatment. Egr-1 expression was higher in TE2 cells compared with other esophageal cancer cell lines, and its knockdown increased 4E-BP1 expression in TE2 cells, which became sensitive to rapamycin treatment.
\end{abstract}

Conclusions: The 4E-BP1/eIF4E ratio is a determinant of the response of rapamycin treatment in esophageal cancer cells. Egr-1 can reduce $4 E-B P 1$ gene expression and render esophageal squamous cell carcinoma cells resistant to rapamycin with a relatively low 4E-BP1/eIF4E ratio. Thus, the 4E-BP1/eIF4E ratio may represent a therapeutic index for the prediction of clinical outcome of rapamycin treatment in patients with esophageal squamous cell carcinoma. (J Thorac Cardiovasc Surg 2015;149:378-85)

See related commentary on pages 386-7.

Esophageal squamous cell carcinoma (ESCC) is associated with poor survival despite surgical resection. Many clinicopathologic variables, including the depth of tumor invasion, lymph node involvement, lymphovascular invasion,

From the Institute of Emergency and Critical Care Medicine, ${ }^{a}$ National Yang-Ming University School of Medicine, Taipei, Taiwan; Division of Thoracic Surgery, ${ }^{\mathrm{b}}$ Department of Surgery, Taipei Veterans General Hospital, Taipei, Taiwan; Department of Biomedical Imaging and Radiological Sciences, ${ }^{c}$ National Yang-Ming University, Taipei, Taiwan; Division of Nuclear Medicine, ${ }^{\mathrm{d}}$ Taipei City Hospital Zhongxiao Branch, Taipei, Taiwan; Institute of Biochemistry and Molecular Biology, ${ }^{\mathrm{e}}$ National Yang-Ming University, Taipei, Taiwan; and Institute of Clinical Medicine ${ }^{\mathrm{f}}$ National Yang-Ming University School of Medicine, Taipei, Taiwan.

Han-Shui Hsu received grants from Taipei Veterans General Hospital (V100C-063, V101C-088, V101E1-016, V102C-028, and V103E8-004) and was supported partly by the Lung Cancer Foundation in Memory of Dr K. S. Lu. Tzu-Hao Cheng received grants from the National Science Council (97-2320-B-010-025 and 102-2325-B-010-017) and the "Aim for the Top University Plan" grant from the Ministry of Education of the Republic of China.

Disclosures: Authors have nothing to disclose with regard to commercial support.

Received for publication June 10, 2014; revisions received Sept 9, 2014; accepted for publication Sept 12, 2014; available ahead of print Oct 23, 2014.

Address for reprints: Tzu-Hao Cheng, $\mathrm{PhD}$, Institute of Biochemistry and Molecular Biology, National Yang-Ming University, No. 155, Sec. 2, Li-Nong St, Taipei,

Taiwan (E-mail: thcheng@ym.edu.tw).

0022-5223/\$36.00

Copyright (c) 2015 by The American Association for Thoracic Surgery

http://dx.doi.org/10.1016/j.jtcvs.2014.09.047 intramural metastasis, and stage of disease, have been examined to predict the prognosis. Multiple molecular changes also have been investigated to elucidate the mechanism of ESCC tumorigenesis, ${ }^{1}$ resulting in the emergence of new therapeutic strategies, including those that target the mammalian target of rapamycin (mTOR) pathway. ${ }^{2}$

mTOR is a serine-threonine kinase belonging to the phosphoinositide 3-kinase-related kinase family.,4 Aberrant activation of the mTOR signaling pathway has been reported in multiple human cancers, including esophageal cancer. ${ }^{5}$ Rapamycin, a Food and Drug Administration-approved antibiotic and immunosuppressant that targets mTOR, is currently used as an anti-tumor agent against ESCC. ${ }^{3}$ Rapamycin inhibits the kinase activity of mTOR, leading to hypophosphorylation of 4E-BP1, enhancement of 4E-BP1 binding competency to eIF4E, and suppression of eIF4E-dependent gene translation. ${ }^{6}$ eIF4E is a cap-binding protein involved in gene translation via a trimetric complex formation with eIF4G and eIF4A, termed "eIF4F." Hypophosphorylated 4E-BP1, as a result of mTOR inhibition, competes with eIF4G in eIF4E binding and prevents the formation of the translation initiation complex, eIF4F. ${ }^{7}$ Although eIF4E has been considered a general translational component, the protein translation of many gene products associated with oncogenesis is sensitive to eIF4E activity, ${ }^{8}$ and it 


\section{Abbreviations and Acronyms \\ ESCC = esophageal squamous cell carcinoma \\ $\mathrm{mTOR}=$ mammalian target of rapamycin \\ $\mathrm{m}^{7} \mathrm{GTP}=7$-Methyl GTP \\ shRNA $=$ short hairpin RNA}

is a potential molecular marker in human cancers. ${ }^{9}$ We have previously found that eIF4E was associated with nodal metastasis in esophageal cancer, and that the protein translation of the oncogene, MDM2, was regulated by eIF4E in tumor tissues. ${ }^{10}$

Rapamycin elicits its anti-tumor effect by suppressing cell cycle progression from $\mathrm{G} 1$ to $\mathrm{S}$ phase, but such cellular effects could be overcome by genetic or epigenetic alterations. ${ }^{11}$ Relative 4E-BP1 versus eIF4E level has been hypothesized as a determinant for the response of cells to rapamycin, ${ }^{12}$ because sequestration of eIF4E by $4 \mathrm{E}-\mathrm{BP} 1$ is necessary for the inhibitory action of rapamycin. In this instance, low 4E-BP1 protein levels were observed in rhabdomyosarcoma or colon carcinoma cells with acquired or intrinsic resistance to rapamycin. Such cellular response was markedly reversed by an increase of 4E-BP1 expression devoid of changes in eIF4E protein level, suggesting that the amount of 4E-BP1 available to sequester eIF4E or the $4 \mathrm{E}-\mathrm{BP} 1 / \mathrm{eIF} 4 \mathrm{E}$ ratio is likely to dictate the cellular response to rapamycin. This notion was further illustrated recently using active-site mTOR inhibitors. ${ }^{13}$ However, analysis of breast cancer cells did not find an association between rapamycin sensitivity and 4E-BP1/eIF4E ratio. ${ }^{14}$ It is unknown whether the relationship between eIF4E and 4E-BP1 could affect the response to rapamycin treatment in ESCC, although the growth inhibition of ESCC by rapamycin has been broadly investigated. ${ }^{15}$

In this study, we demonstrated that aberrant $4 E-B P 1$ gene expression is caused by transcription suppression of Egr-1, and rapamycin response of ESCC is determined by the 4E-BP1/eIF4E ratio. Cells having a low 4E-BP1/eIF4E ratio are resistant to rapamycin treatment, and the cellular response can be revered by 4E-BP1 overexpression or eIF4E short hairpin RNA (shRNA) knockdown. Conversely, cells that were originally responsive to rapamycin become insensitive on ectopic overexpression of eIF4E or 4E-BP1 knockdown. Thus, the 4E-BP1/eIF4E ratio could be a reliable marker to predict the efficacy of rapamycin in ESCC.

\section{MATERIALS AND METHODS \\ Cell Culture}

The ESCC cell lines CE48T/VGH, CE81T/VGH, CE146T, HCE8, TE1, and TE2 were provided by Dr F. H. Wong. ${ }^{16}$ Cells were cultured in
Dulbecco's modified Eagle's medium supplemented with $10 \%$ fetal bovine serum at $37^{\circ} \mathrm{C}$ with $5 \% \mathrm{CO}_{2}$.

\section{Rapamycin Treatment}

ESCC cells were seeded in 96-well plates, cultured in growth medium for 1 day, and switched to serum-free medium to enhance the cellular response of rapamycin. Cells were then washed twice with warm phosphate-buffered saline $(10 \mathrm{mmol} / \mathrm{L}$ sodium phosphate $\mathrm{pH} 7.4$ and $0.9 \% \mathrm{NaCl}$ ) and cultured in fresh Dulbecco's modified Eagle's medium containing various concentrations of rapamycin dissolved in dimethyl sulfoxide (Sigma, St Louis, Mo) with $2 \%$ fetal bovine serum. After 5 days, cell viability was determined using the MTT assay as described previously. ${ }^{17}$

To analyze the content of eIF4E-bound proteins on rapamycin treatment, cells were treated with $1 \mu \mathrm{mol} / \mathrm{L}$ rapamycin or dimethyl sulfoxide (vehicle control) in 10-cm dishes for 24 hours and then subjected to the 7-Methyl GTP ( $\left.\mathrm{m}^{7} \mathrm{GTP}\right)$ pull-down assay, as described next.

\section{7-Methyl GTP Pull-Down Assay}

The eIF4E-containing protein complex was detected using affinity chromatography with $\mathrm{m}^{7}$ GTP-sepharose as previously described. ${ }^{18}$ In brief, after rapamycin treatment, ESCC cells were resuspended in cap binding buffer $(10 \mathrm{mmol} / \mathrm{L}$ sodium phosphate $\mathrm{pH} 7.5,100 \mathrm{mmol} / \mathrm{L} \mathrm{KCl}$, $1 \mathrm{mmol} / \mathrm{L}$ dithiothreitol, $2 \mathrm{mmol} / \mathrm{L} \mathrm{MgCl}_{2}, 1 \mathrm{mg} / \mathrm{mL}$ RNase, and $1 \mathrm{X}$ complete protease inhibitor). After sonication to lyse the cells, the supernatant was collected and mixed with $\mathrm{m}^{7} \mathrm{GTP}$-sepharose resin (Amersham Bioscience, Piscataway, NJ). In each sample, $500 \mu \mathrm{g}$ of cell lysate was incubated with $30 \mu \mathrm{L}$ of resin at $4^{\circ} \mathrm{C}$ for 1 hour and then washed 3 times with a wash buffer consisting of cap binding buffer with $0.5 \%$ NP-40. The protein complexes, which contain eIF4E and its interacting proteins, were removed from the resin by boiling in sodium dodecyl sulfate loading buffer and analyzed by Western blotting.

\section{Western Blot Analysis}

Western blot analysis was performed as described previously ${ }^{19}$ using antibodies against $\beta$-actin (AC-15, Abcam, Cambridge, Mass), eIF4E (610270, BD Biosciences, San Jose, Calif), 4E-BP1 (GTX22606, GeneTex, San Antonio, Tex), eIF4G (610536, BD Biosciences), Egr-1 (44D5, Cell Signaling, Beverly, Mass), and murine double minute 2 (MDM2; 4B11, Calbiochem, San Diego, Calif).

\section{Flow Cytometry}

Cells were fixed in $70 \%$ ethanol at $-20^{\circ} \mathrm{C}$ for 16 hours, after which they were treated with RNase $\mathrm{A}(1 \mathrm{mg} / \mathrm{mL})$ at $37^{\circ} \mathrm{C}$ for 15 minutes, followed by staining with propidium iodide $(50 \mu \mathrm{g} / \mathrm{mL})$ at $37^{\circ} \mathrm{C}$ for an additional $15 \mathrm{mi}-$ nutes. For cell cycle analysis, these samples were analyzed by flow cytometry using a FACScan system (BD Biosciences), and data were processed with WinMDI software.

\section{Lentivirus Preparation and Infection}

Because TE1 and TE2 cells have an extremely low nucleic acid transfection efficiency using commercialized transfection reagents, they were infected by lentivirus carrying different shRNA or gene-coding DNA sequences in this study. Empty viral vectors (TRC1 and TRC2) and those expressing shRNA against human eIF4E, 4E-BP1, or Egr-1 were obtained from the National RNAi core (Academia Sinica, Taipei, Taiwan). Two individual shRNA constructs of eIF4E (TRCN 0000062573 and 0000062574), 4E-BP1 (TRCN 0000040203 and 0000040206), or Egr-1 (TRCN 0000273850 and 0000273851) were used for gene silencing. Lentiviral transduction particles were prepared as described and used to transduce esophageal cancer cell lines. ${ }^{20}$ Enforced expression of 4E-BP1 or eIF4E was carried out after the coding sequence of these genes was 


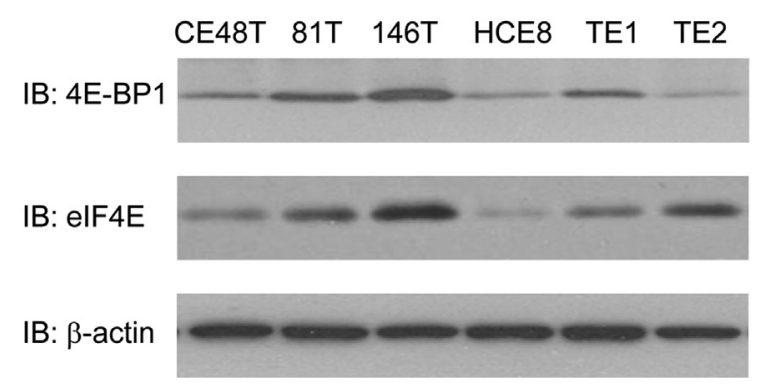

A

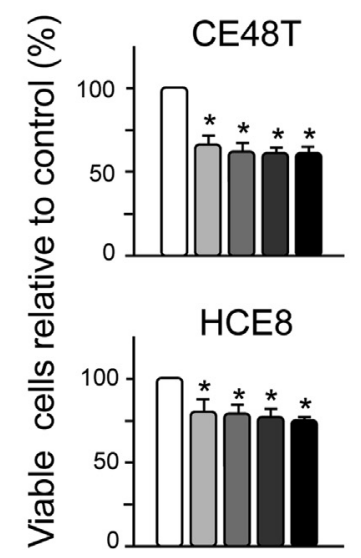

TE1
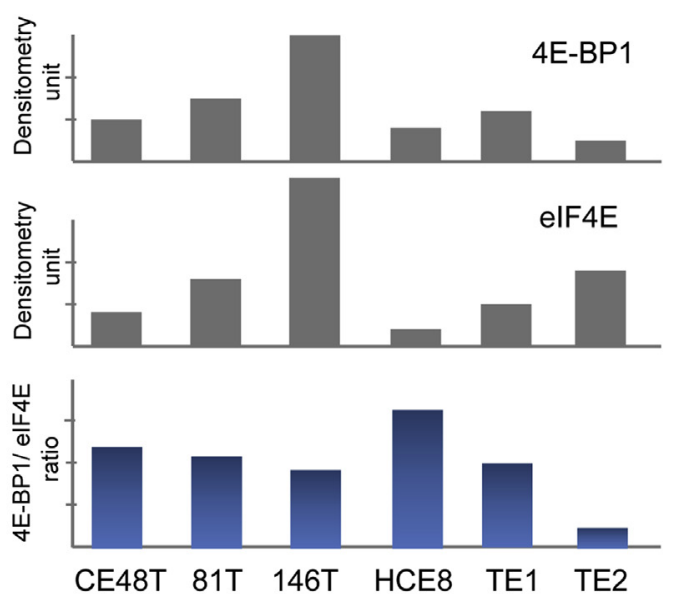
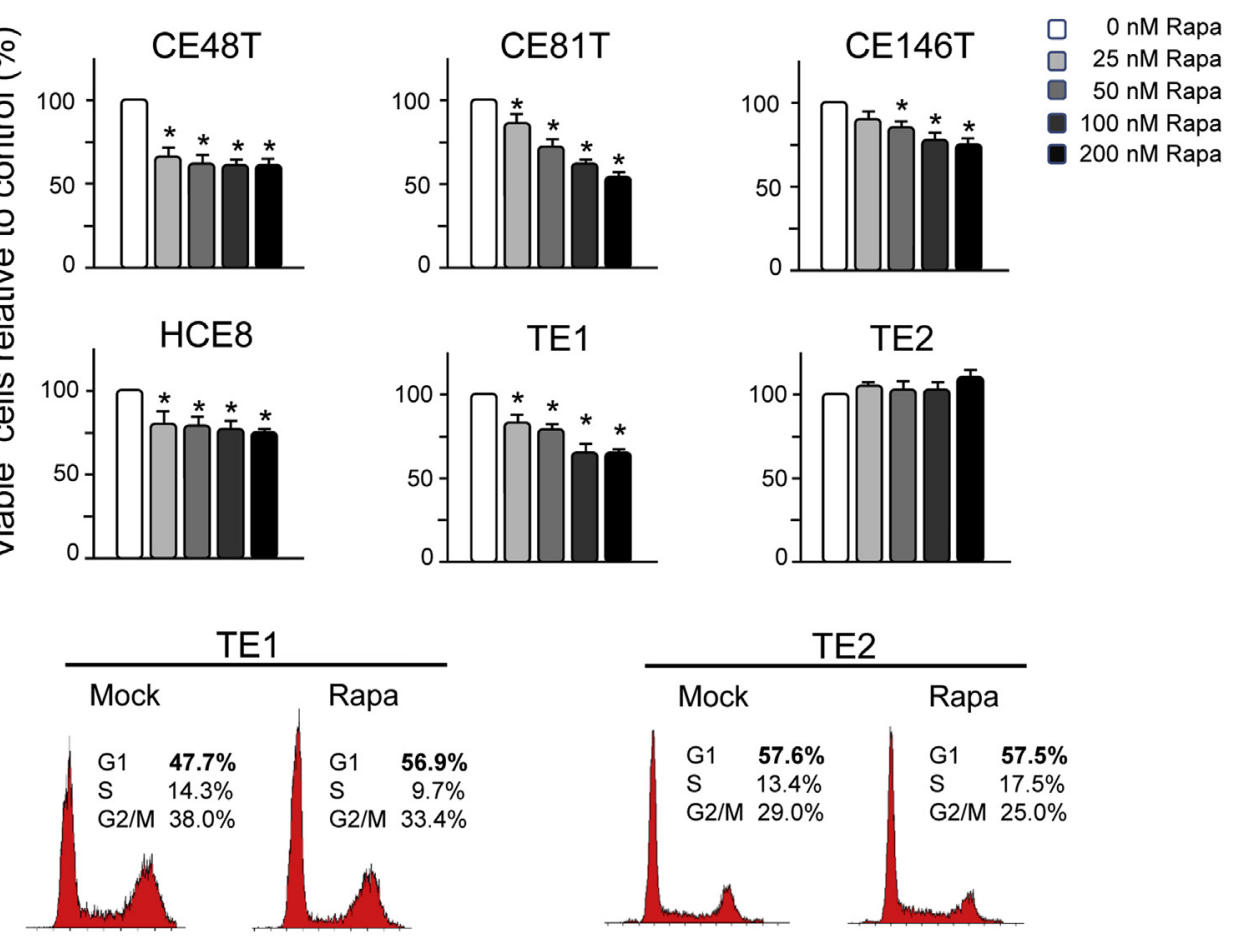

B

C

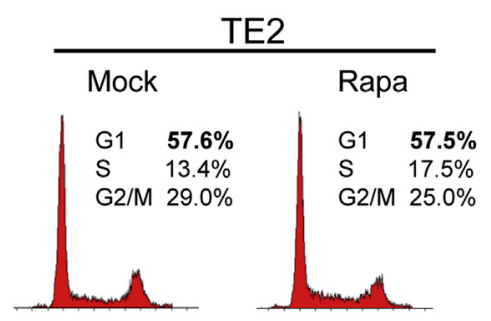

FIGURE 1. Rapamycin insensitivity is associated with a relatively low 4E-BP1/eIF4E ratio in ESCC cell lines. A, 4E-BP1 and eIF4E protein levels were assessed by Western blot analysis in ESCC cell lines (left). Protein expression was quantified by densitometry, and the ratio of 4E-BP1 versus eIF4E in each sample is shown $(r i g h t) . \beta$-actin serves as an internal loading control. B, Cells were treated with different doses of rapamycin, and viable cells were measured using the MTT assay. Samples without rapamycin treatment were set as $100 \%$, and relative viable cells to the control are shown. Results were the average of 3 independent experiments done in hexaplicate $(* P<.05$, Student $t$ test). C, TE1 and TE2 cells were stained with propidium iodide and analyzed by flow cytometry after the treatment of rapamycin $(1 \mu \mathrm{mol} / \mathrm{L}$ for 24 hours $)$ or mock control.

subcloned from CMV-eIF4E or pACTAG-4E-BP1 into a pLenti4-based lentiviral vector for cytomegalovirus promoter-driven transgene overexpression. Viruses were prepared, and ESCC cells were infected as described earlier.

\section{Reverse Transcription-Polymerase Chain Reaction}

As described previously, ${ }^{19}$ an equivalent amount of total RNA from each sample was converted into complementary DNA and polymerase chain reaction amplified using the following primer sets: 4E-BP1 sense, $5^{\prime}$-GAGAG GTTCGCGGGTGCAG- $3^{\prime}$ and antisense, $5^{\prime}$-CCAACGCCTGCCCAGT ATG $-3^{\prime}$; and $\beta$-actin, sense $5^{\prime}$-AAGTACTCCGTGTGGATCGG- $3^{\prime}$ and antisense $5^{\prime}$-ATGCTATCACCTCCCCTGTG- $3^{\prime} . \quad \beta$-actin served as an internal control.

\section{RESULTS}

\section{E-BP1/eIF4E Ratio Is Associated With Esophageal Cancer Cell Response to Rapamycin}

To investigate the factor(s) that govern cellular response to rapamycin in ESCC, 4E-BP1 and eIF4E protein levels were examined in 6 different esophageal cancer cell lines. 
Although the quantity of 4E-BP1 or eIF4E varies among these samples, it is evident that TE2 cells have a relatively low 4EBP1/eIF4E ratio (Figure 1, A). These cells were treated with rapamycin concentrations ranging from 25 to $200 \mathrm{nM}$, and the effects on growth were analyzed. Most cell lines, including TE1, had a substantial decrease in the number of viable cells on treatment with $200 \mathrm{nM}$ rapamycin. However, there was no significant change or even a slight increase of viable TE2 cells cultured in the same condition (Figure 1, B).

Because rapamycin inhibits cell cycle progression and reduces cell proliferation, ${ }^{21}$ rapamycin-treated cells were further examined using flow cytometry. Moderate accumulation of TE1 cells in G1 phase was detected with rapamycin; however, TE2 cells experienced no obvious change in cell cycle distribution (Figure 1,C), indicating that TE2 cells were insensitive to rapamycin. Unlike the other esophageal cancer cell lines examined, TE2 cells had a relatively low 4E-BP1/ eIF4E ratio, suggesting the cellular response to rapamycin may likely be dependent on 4E-BP1/eIF4E ratio.

\section{Altered 4E-BP1/eIF4E Ratio Changes Rapamycin Sensitivity in Esophageal Cancer Cells}

To evaluate the significance of the 4E-BP1/eIF4E ratio in the response of ESCC cells to rapamycin, the protein level of 4E-BP1 or eIF4E was adjusted in TE1 and TE2 esophageal cancer cells. Both cell lines showed an efficient ectopic gene expression and shRNA knockdown by viral transduction system. After ectopic 4E-BP1 overexpression, TE2 cells changed their cellular response to rapamycin from insensitive to sensitive (Figure 2, A). TE2 cells also became sensitive to rapamycin treatment after knockdown of eIF4E (Figure 2, $B$ ). In both cases, the 4E-BP1/eIF4E ratio was increased, suggesting that a high 4E-BP1/eIF4E ratio is necessary for rapamycin to execute its cellular effect. In agreement with this idea, TE1 cells became resistant to rapamycin treatment after the 4E-BP1/eIF4E ratio decreased on 4E-BP1 knockdown (Figure 2,C) or eIF4E overexpression (Figure 2,D). Thus, the ratio of $4 \mathrm{E}-\mathrm{BP} 1 / \mathrm{eIF} 4 \mathrm{E}$ is a key determinant for the response of esophageal cancer cells to rapamycin treatment.

\section{eIF4F Translation Initiation Complex and MDM2 Protein Expression Are Not Affected by Rapamycin in TE2 Cells}

The eIF4F translation initiation complex, containing eIF4E and eIF4G, is involved in the rate-limiting step of protein synthesis. ${ }^{22}$ Rapamycin can impede protein translation by promoting the dephosphorylation of $4 \mathrm{E}-\mathrm{BP} 1$, which sequesters eIF4E from eIF4G and prevents the formation of eIF4F. Several oncogenes, such as cyclin D1 and MDM2, are more dependent on eIF4F activity, and the protein synthesis of these genes is substantially reduced by rapamycin treatment. ${ }^{19}$ To evaluate eIF4F status in rapamycin-treated cells, $m^{7}$ GTP pull-down assays were performed using TE1 and TE2 cells. In TE1 cells, eIF4E was mainly occupied by $4 \mathrm{E}-\mathrm{BP} 1$ on rapamycin treatment; however, this does not occur in TE2 cells (Figure 3, A). Although 4E-BP1 was dephosphorylated in both cell lines, the protein was not able to compete with the eIF4G in the translation initiation complex of TE2 cells that have a low 4E-BP1/eIF4E ratio. Accordingly, the protein synthesis of MDM2 was not downregulated by rapamycin in TE2 cells (Figure 3, B).

\section{Egr-1 Upregulation Contributes to Rapamycin Insensitivity in TE2 Cells}

Egr-1, a zinc finger transcription factor involved in diverse mitogenic signals, can repress the promoter activity of $4 E-B P 1 .{ }^{23}$ To investigate the role of Egr-1 in the regulation of 4E-BP1 in esophageal cancer cells, Western blot analysis was first performed. As shown in Figure 4, A, Egr-1 protein level was higher in TE2 cells compared with other esophageal cancer cell lines. After Egr-1 shRNA knockdown (Figure 4,C), $4 E$-BP1 mRNA was increased in TE2 cells (Figure 4, $B$ ). As described in Figure 2, $A$ and $B$, Egr-1 shRNA-treated cells with 4E-BP1 induction and consequent increase in 4E-BP1/eIF4E ratio also became rapamycin-sensitive (Figure 4, C). Overall, these experiments suggested that Egr-1 is responsible, at least in part, for the aberrant gene repression of $4 E-B P 1$, low 4E-BP1/eIF4E ratio, and rapamycin insensitivity of TE2 cells.

\section{DISCUSSION}

By using several esophageal cancer cell lines, we demonstrated that the relative 4E-BP1/eIF4E ratio, rather than their individual protein levels, determines the efficacy of rapamycin treatment in ESCC cells. In addition, Egr-1 can modulate the mRNA expression of $4 E-B P 1$, thereby influencing the $4 \mathrm{E}-\mathrm{BP} 1 / \mathrm{eIF} 4 \mathrm{E}$ ratio and consequently determining the cellular response to rapamycin. These experimental results are summarized in Table 1, and a schematic model showing the molecular action of rapamycin and the loss of rapamycin sensitivity in esophageal cancer cells that are caused by a low 4E-BP1/eIF4E protein ratio is illustrated in Figure 5.

Although a previous study reported that eIF4E overexpression was associated with tumor progression and described a possible role for 4E-BP1 as a prognostic factor in esophageal cancer, ${ }^{24}$ the contribution of $4 \mathrm{E}-\mathrm{BP} 1$ or eIF4E to rapamycin response was unclear. Grosso and colleagues ${ }^{25}$ recently found that downregulation of eIF4E and overexpression of 4E-BP1 can induce rapamycin sensitivity in mesothelioma cells. In the present in vitro study, rapamycin sensitivity of TE1 cells decreased when the ratio of 4E$\mathrm{BP} 1 / \mathrm{eIF} 4 \mathrm{E}$ was decreased by reducing 4E-BP1 expression or eIF4E overexpression. Conversely, increasing the 4EBP1/eIF4E ratio with 4E-BP1 overexpression or eIF4E knockdown increased TE2 cell sensitivity to rapamycin. Because rapamycin response is associated with intrinsic 
A

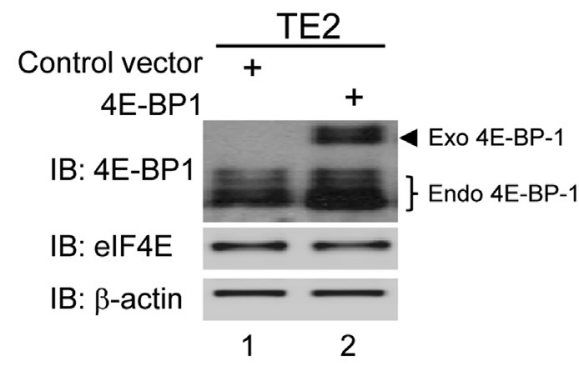

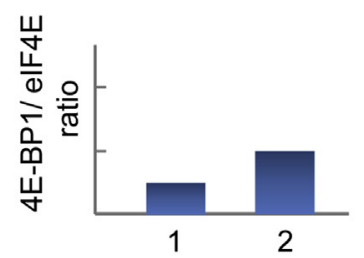

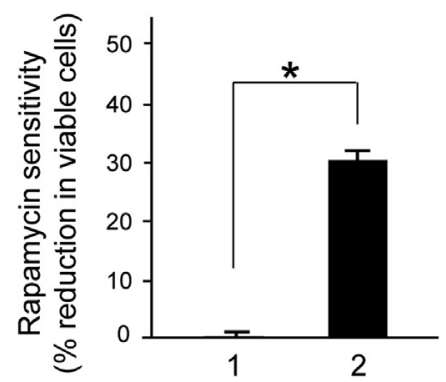

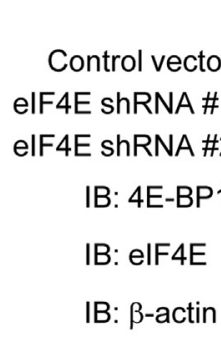

B
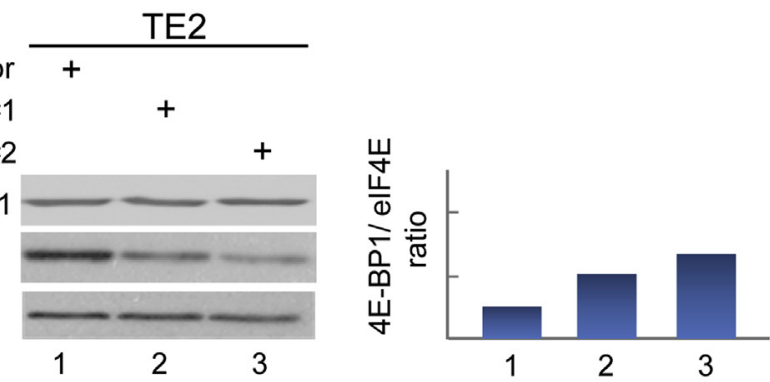

\section{A

$\# 1$

TE1

4E-BP1 shRNA \#1
4E-BP1 shRNA \#2
IB: 4E-BP1
IB: elF4E
IB: $\beta$-actin

C
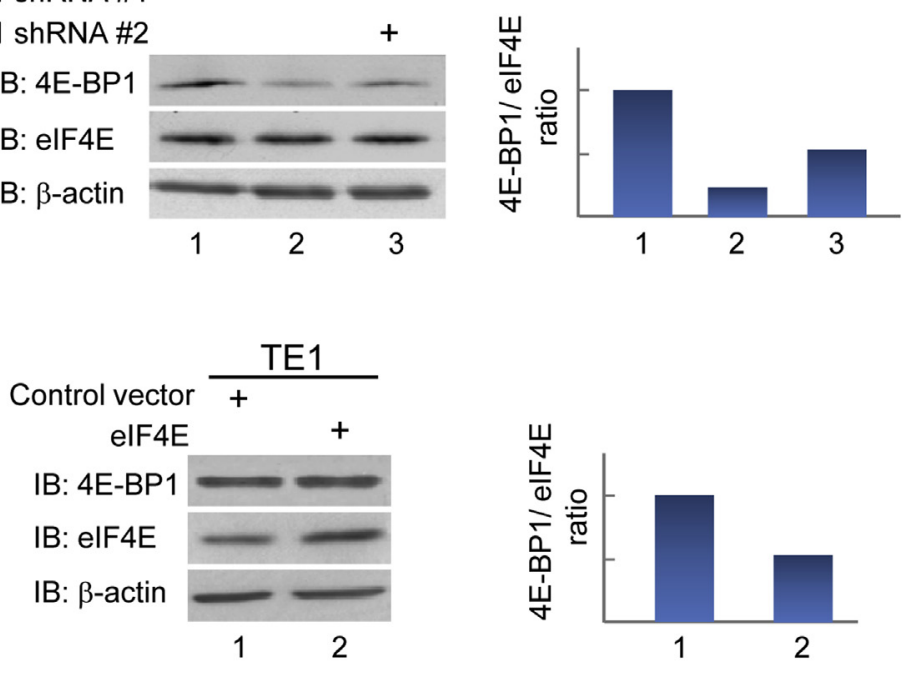
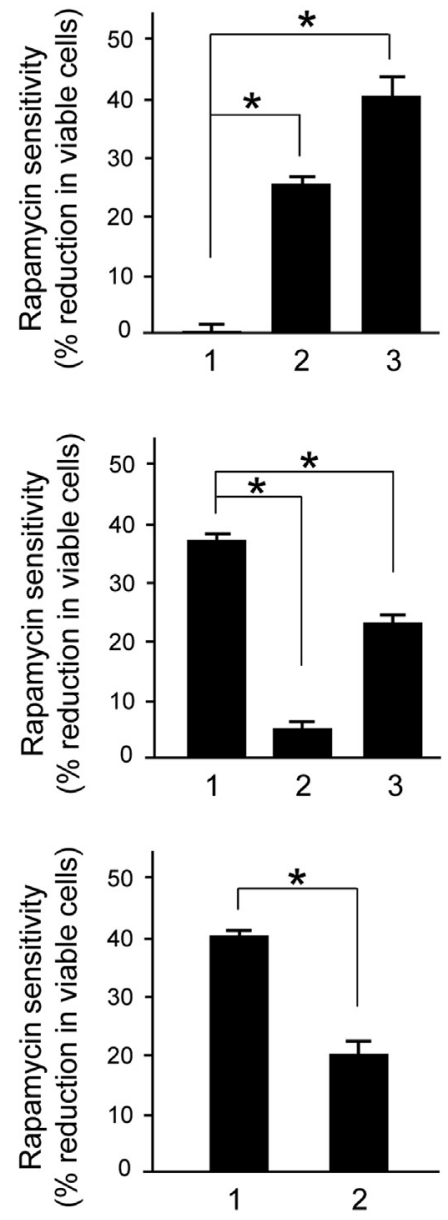

FIGURE 2. Changes in 4E-BP1/eIF4E ratio redirect esophageal cancer cell response to rapamycin. A, TE2 cells become sensitive to rapamycin treatment on 4E-BP1 overexpression. TE2 cells were infected with the empty viral vector or the construct expressing hemagglutinin (HA)-tagged 4E-BP1. The protein levels of 4E-BP1 and eIF4E were examined by Western blot analysis. Endogenous and exogenous 4E-BP1 are indicated, and $\beta$-actin serves as a loading control (left). The 4E-BP1/eIF4E ratio was quantified as described in Figure 1, A (middle). Rapamycin sensitivities are shown and represent the \% reductions of viable cells caused by $200 \mathrm{nM}$ rapamycin as described in Figure 1, B. Data presented are means \pm standard deviation of 3 independent experiments $\left({ }^{*} P<.05\right.$, Student $t$ test) $(r i g h t)$. B, eIF4E knockdown with lentivirus expressing specific shRNAs against eIF4E, resulting in increased 4E-BP1/eIF4E ratio, also increases TE2 cell sensitivity to rapamycin. C, TE1 cells change from rapamycin-sensitive to insensitive after 4E-BP1 knockdown using lentivirus that expresses 4E-BP1 shRNAs. D, TE1 cells become insensitive to rapamycin treatment when the 4E-BP1/eIF4E ratio is decreased by eIF4E overexpression using a viral construct expressing eIF4E. shRNA, Short hairpin RNA.

4E-BP1/eIF4E ratio and adjustment of this protein ration can change the cellular response accordingly, our results suggest that the 4E-BP1/eIF4E ratio is a determinant for the efficacy of rapamycin treatment in ESCC.
In this investigation, we found that TE2 cells had the lowest 4E-BP1/eIF4E ratio and were resistant to rapamycin treatment. The other 5 cell lines with a relatively higher 4E$\mathrm{BP} 1 / \mathrm{eIF} 4 \mathrm{E}$ ratio were all response to rapamycin treatment; 


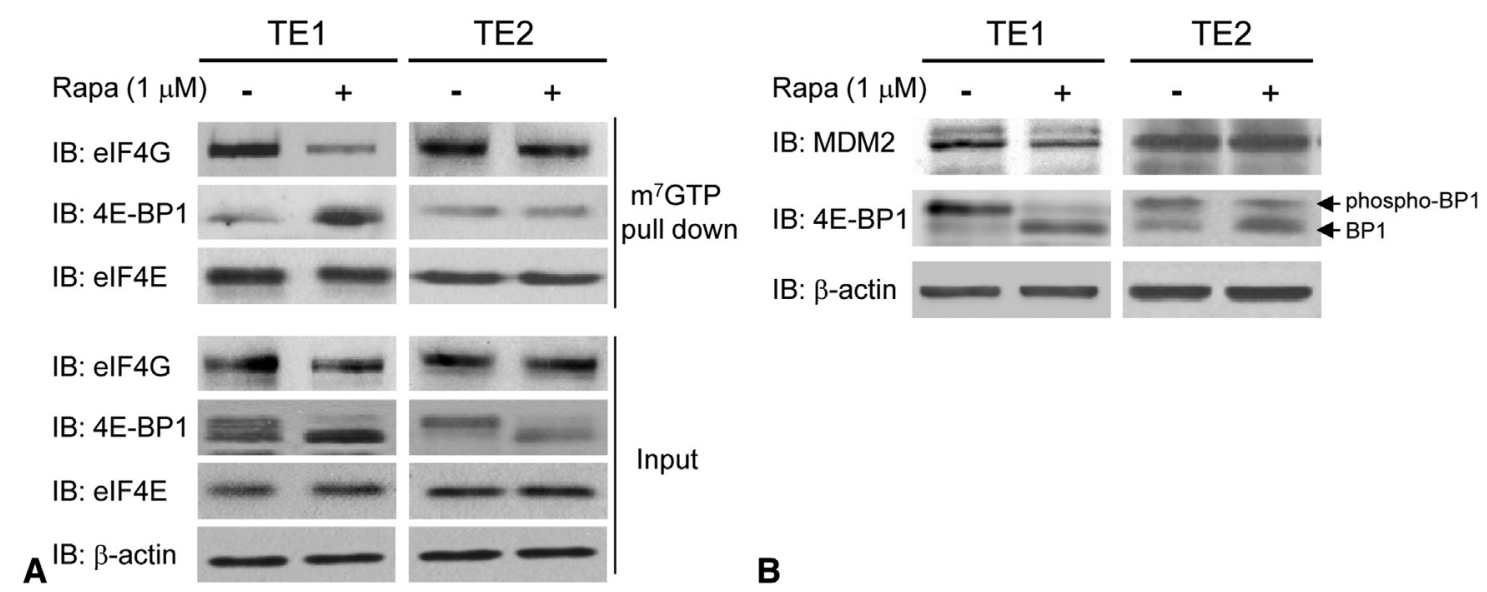

FIGURE 3. The assembly of eIF4E with eIF4G is interrupted by 4E-BP1 on rapamycin treatment in TE1 cells, but not in TE2 cells. A, The indicated cells were treated with $1 \mu \mathrm{mol} / \mathrm{L}$ rapamycin for 24 hours. Total cell lysates were prepared and subjected to $\mathrm{m}^{7} \mathrm{GTP}$ pull-down assay, followed by Western blot analysis to detect eIF4E, eIF4G, and 4E-BP1. B, MDM2 protein synthesis was examined by Western blot analysis using cell lysates as described in (A). Rapamycin inhibits 4E-BP1 protein phosphorylation, and the isoforms (phosphor-BP1 and BP1) are indicated. $\beta$-actin served as a loading control. $m^{7} G T P, 7-M e t h y l$ GTP.

however, the extent of response as reflected by cell growth inhibition varied. In addition, HCE8 cells had the highest 4EBP1/eIF4E ratio, but the response to rapamycin treatment was fair and not as good as the response in TE1 cells. Our findings suggest that the ratio can define the occurrence of rapamycin response, but it may not serve as a predictor for the degree of cellular response, which is likely governed by oncogenic genes that are susceptible to eIF4E and varying in each esophageal cancer cell line.
eIF4E, a translation initiation factor, is associated with multiple human cancers and is selectively required for expression of a subgroup of genes with roles in oncogenesis. ${ }^{26}$ Rapamycin attenuates eIF4E activity via hypophosphorylation of 4E-BP1 as a consequence of mTOR inhibition. Therefore, gene mutations that prevent 4EBP1 hypophosphorylation or trigger a persistent activation of mTOR kinase activity may result in rapamycin resistance. In both rapamycin-sensitive TE1 and rapamycin-
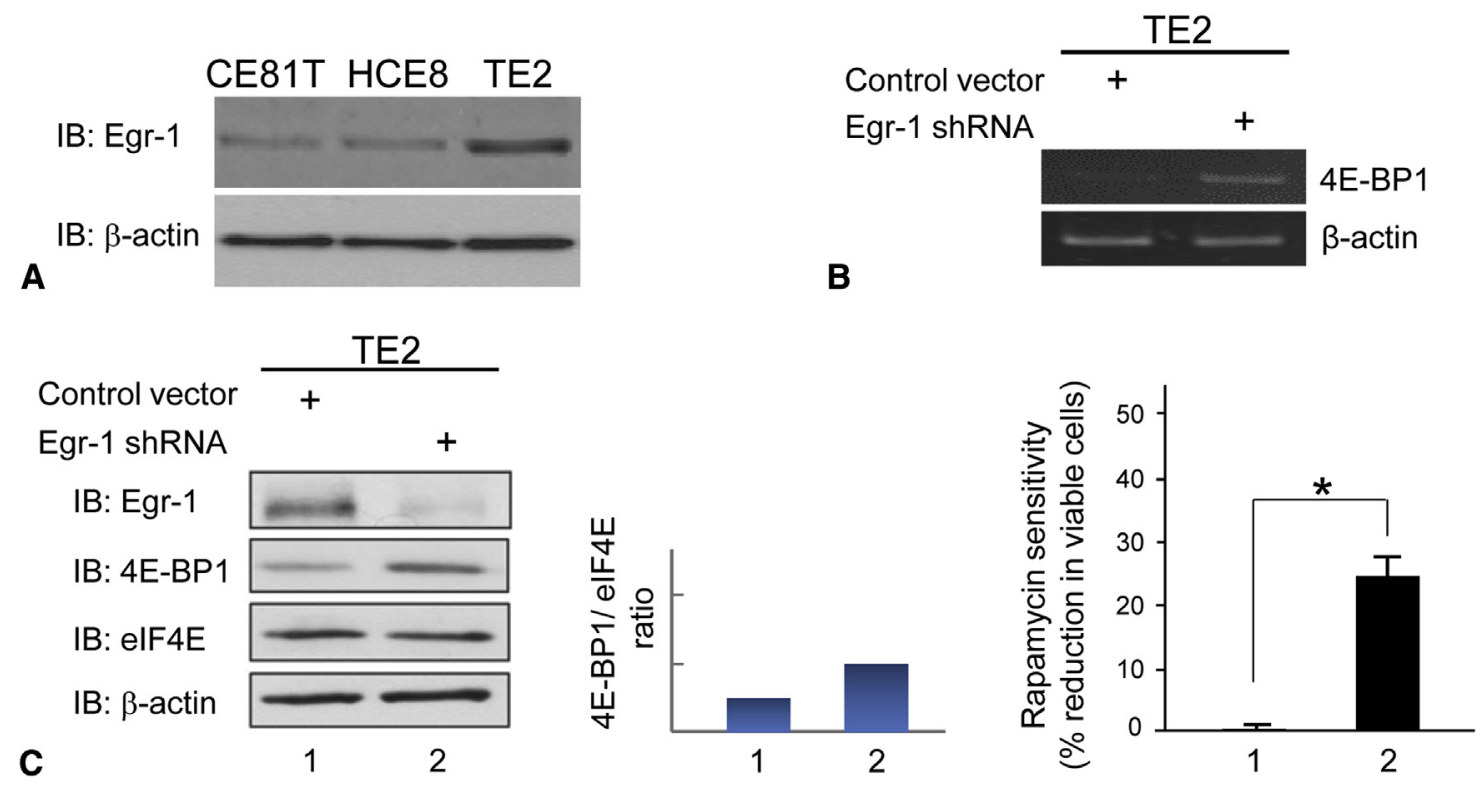

FIGURE 4. Egr-1 contributes the low $4 E-B P 1$ gene expression and rapamycin insensitivity of TE2 cells. A, Lysates of the indicated ESCC cells were collected, and Egr-1 protein levels were examined by Western blot analysis. $\beta$-actin serves as a loading control. B, TE2 cells were subjected to Egr-1 shRNA knockdown, and the change of $4 E-B P 1$ mRNA expression was analyzed by reverse transcriptase-polymerase chain reaction. C, Egr-1, 4E-BP1, and eIF4E protein levels were assessed by Western blot analysis in TE2 cells with or without Egr-1 shRNA knockdown (left). The 4E-BP1/eIF4E ratio as determined by densitometry in each sample is indicated (middle). Rapamycin sensitivity of TE2 cells on Egr-1 knockdown was measured. Data presented are means \pm standard deviation of 3 independent experiments $(* P<.05$, Student $t$ test) (right). shRNA, Short hairpin RNA. 
TABLE 1. Summary of the experimental results of rapamycin treatment in esophageal cancer

\begin{tabular}{lcc}
\hline & \multicolumn{2}{c}{ Cell lines } \\
\cline { 2 - 3 } & TE1 & TE2 \\
\hline 4EBP1/eIF4E ratio & High & Low \\
Treatment with rapamycin & & \\
Rapamycin sensitive & Yes & No \\
Cell cycle arrest & Yes & No \\
eIF4E/eIF4G interaction & Reduced & No change \\
MDM2 protein synthesis & Reduced & No change \\
Expression of Egr-1 & ND & High \\
\hline$N D$, Not determined. & &
\end{tabular}

resistant TE2 cells, 4E-BP1 phosphorylation levels decreased to a similar extent on rapamycin treatment. However, the translation initiation complex eIF4F was disrupted in TE1 cells but not TE2 cells. Thus, the mTOR/4EBP1 axis may not be perturbed in ESCC cells, and a defect in 4E-BP1 hypophosphorylation cannot account for rapamycin resistance. Furthermore, the relative amount of 4E-BP1 plays a central role in occupancy of eIF4E as evidenced by a lack of eIF4F disruption in TE2 cells.

Rapamycin acts as a cytostatic agent, slowing or arresting the growth of cell lines from different tumor types, such as small cell lung and prostate cancers. ${ }^{27,28}$ Our previous study showed that the anti-tumor effect of rapamycin to some extent is caused by translational inhibition of MDM2. ${ }^{19}$ We also found that MDM2 protein levels are strongly associated with and regulated by eIF4E in a post-transcriptional mechanism in esophageal cancer. ${ }^{10}$ Because the protein synthesis of MDM2 was reduced by inactivation of eIF4E in TE1 cells, our data suggested that MDM2 downregulation may have an anti-tumor effect in rapamycin-treated esophageal cancer cells.

4E-BP1, a eIF4E negative regulator, was also found to be associated with several human cancers. For example, O'Reilly and colleagues ${ }^{29}$ showed that phosphorylated 4E-BP1 was associated with poor survival in melanoma. In addition, She and colleagues ${ }^{30}$ reported that combined inhibition of AKT and MEK is feasible and effectively inhibits 4E-BP1 phosphorylation and tumor growth. Although post-translational modification of 4E-BP1 has been the focus many studies, transcriptional regulation of 4E-BP1 also played an essential role for its biological function in esophageal cancer. Specifically, on downregulation of Egr-1, the transcript and protein levels of 4E-BP1 were increased in TE2 cells, accompanied by a change of cellular response to rapamycin. Egr-1 was initially identified as a transcription factor, regulating neuronal differentiation $^{31}$; its aberrant expression was associated with cancer development and progression in human malignancies. ${ }^{32} 4 E-B P 1$ has been identified as an Egr-1 target gene $^{23}$; however, the role of Egr-1 on the expression and function of 4E-BP1 in ESCCs was unknown until this study.

The 4E-BP1/eIF4E ratio in esophageal cancer specimens was examined by immunoblot analysis by Salehi and Mashayekhi. ${ }^{24}$ The authors found that the tumor tissue had a higher 4E-BP1/eIF4E ratio than normal adjacent tissue. However, the prognostic impact of the ratio was not
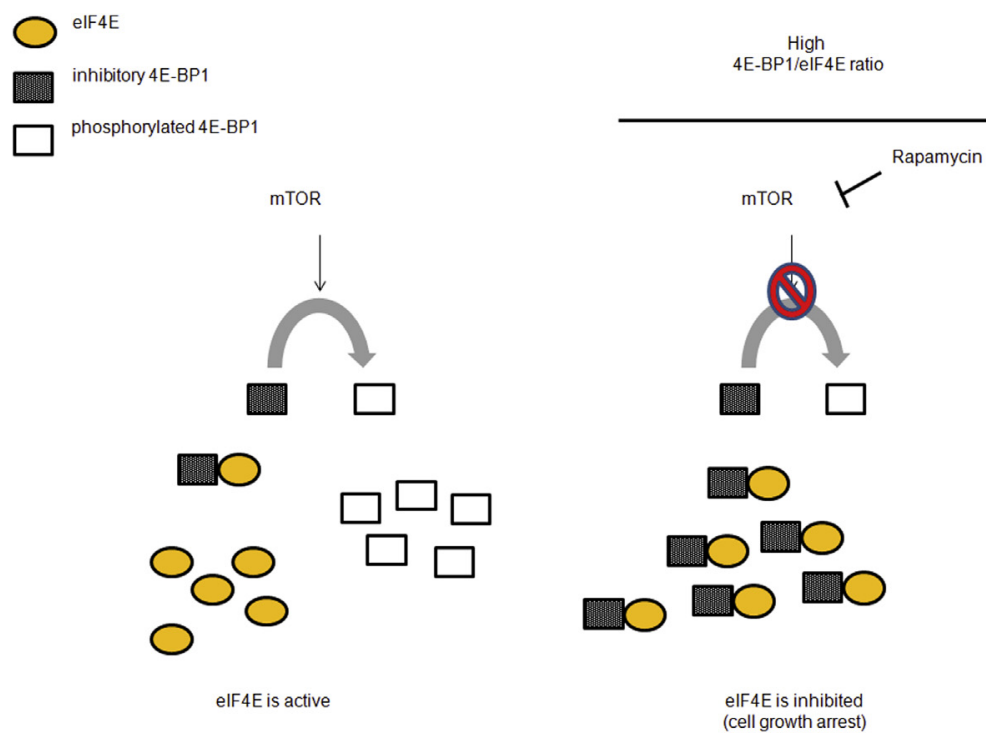

Low
4E-BP1/eIF4E ratio

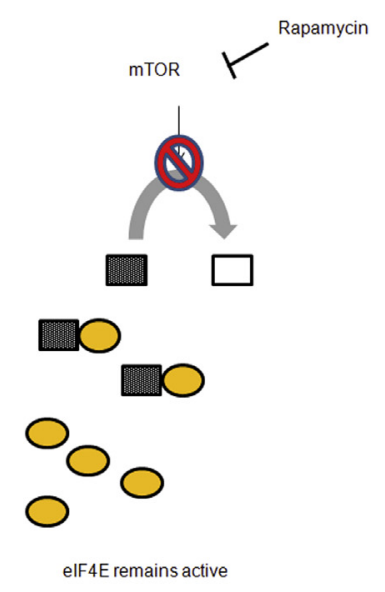

FIGURE 5. Schematic model showing the response of rapamycin is determined by 4E-BP1/eIF4E ratio in esophageal cancer cells. mTOR, a protein complex with kinase activity, phosphorylates 4E-BP1 and makes the protein incapable of binding to and inhibition of eIF4E. When 4E-BP1 phosphorylation is prevented by mTOR inhibitor rapamycin, unphosphorylated 4E-BP1 is accumulated, and consequently the activity of eIF4E that is vital for the growth of esophageal cancers is inhibited. This rapamycin-mediated response is abrogated in cells with a low 4E-BP1/eIF4E ratio, resulting from the deficient amount of 4E-BP1 to sequester eIF4E. mTOR, Mammalian target of rapamycin. 
mentioned in the study. There are few clinical data regarding the treatment effect of rapamycin in patients with esophageal cancer in the literature. In 2010, Okamoto and colleagues ${ }^{33}$ reported shrinkage of metastasis in supraclavicular lymph nodes in a patient with esophageal cancer receiving rapamycin treatment. Recently, in another phase I clinical trial, Werner and colleagues ${ }^{34}$ also reported that antitumor activity was observed in patients with esophagogastric adenocarcinoma receiving combination treatment with rapamycin and mitomycin C. Further study to elucidate the effect of rapamycin therapy in patients with esophageal cancer is warranted.

\section{CONCLUSIONS}

Our study showed for the first time that the ratio of 4E$\mathrm{BP} 1 / \mathrm{eIF} 4 \mathrm{E}$ is a critical determinant to the response of ESCC cells to rapamycin treatment. Thus, the 4E-BP1/ eIF4E ratio in the tumor could serve as a predictive marker for the efficacy of rapamycin and may be useful in identifying patients with ESCC who will most benefit from rapamycin treatment alone or combined with other chemotherapies.

The authors thank Drs Fen-Hwa Wong and Vitaly A. Polunovsky for providing experimental materials and Kelly Su for secretarial assistance. This work was assisted by the Division of Experimental Surgery of the Department of Surgery, Taipei Veterans General Hospital, and Lung Cancer Foundation in Memory of Dr K. S. Lu.

\section{References}

1. Hsu PK, Li AFY, Wang YC, Hsieh CC, Huang MH, Hsu WH, et al. Reduced membranous $\beta$-catenin protein expression is associated with metastasis and poor prognosis in squamous cell carcinoma of the esophagus. J Thorac Cardiovasc Surg. 2008;135:1029-35.

2. Mohamed A, El-Rayes B, Khuri FR, Saba NF. Targeted therapies in metastatic esophageal cancer: advances over the past decade. Crit Rev Oncol Hematol. 2014;91:186-96.

3. Populo H, Lopes JM, Soares P. The mTOR signalling pathway in human cancer. Int J Mol Sci. 2012;13:1886-918.

4. Shaw RJ, Cantley LC. Ras, PI(3)K and mTOR signalling controls tumour cell growth. Nature. 2006;441:424-30.

5. Hirashima K, Baba Y, Watanabe M, Karashima RI, Sato N, Imamura Y, et al. Aberrant activation of the mTOR pathway and anti-tumour effect of everolimus on esophageal squamous cell carcinoma. Br J Cancer. 2012;106:876-82.

6. Yellen P, Saqcena M, Salloum D, Feng J, Preda A, Xu L, et al. High-dose rapamycin induces apoptosis in human cancer cells by dissociating mTOR complex 1 and suppressing phosphorylation of 4E-BP1. Cell Cycle. 2011;10:3948-56.

7. Gingras AC, Raught B, Sonenberg N. eIF4 initiation factors: effectors of mRNA recruitment to ribosomes and regulators of translation. Annu Rev Biochem. 1999; 68:913-63.

8. Avdulov S, Li S, Michalek V, Burichter D, Peterson M, Perlman DM, et al. Activation of translation complex eIF4F is essential for the genesis and maintenance of the malignant phenotype in human mammary epithelial cells. Cancer Cell. 2004; 5:553-63.

9. De Benedetti A, Graff JR. eIF-4E expression and its role in malignancies and metastases. Oncogene. 2004;23:3189-99.

10. Hsu HS, Chen HW, Kao CL, Wu ML, Li AFY, Cheng TH. MDM2 is overexpressed and regulated by the eukaryotic translation initiation factor $4 \mathrm{E}$ (eIF4E) in human squamous cell carcinoma of esophagus. Ann Surg Oncol. 2011;18: 1469-77.

11. Kurmasheva RT, Huang S, Houghton PJ. Predicted mechanisms of resistance to mTOR inhibitors. Br J Cancer. 2006;95:955-60.

12. Dilling MB, Germain GS, Dudkin L, Jayaraman AL, Zhang X, Harwood FC, et al. 4E-binding proteins, the suppressors of eukaryotic initiation factor $4 \mathrm{E}$, are down-regulated in cells with acquired or intrinsic resistance to rapamycin. J Biol Chem. 2002;277:13907-17.

13. Alain T, Morita M, Fonseca BD, Yanagiya A, Siddiqui N, Bhat M, et al. eIF4E/ 4E-BP ratio predicts the efficacy of mTOR targeted therapies. Cancer Res. 2012; 72:6468-76.

14. Noh WC, Mondesire WH, Peng J, Jian W, Zhang H, Dong J, et al. Determinants of rapamycin sensitivity in breast cancer cells. Clin Cancer Res. 2004;10:1013-23.

15. Hou G, Xue L, Lu Z, Fan T, Tian F, Xue Y. An activated mTOR/p70S6K signaling pathway in esophageal squamous cell carcinoma cell lines and inhibition of the pathway by rapamycin and siRNA against mTOR. Cancer Lett. 2007;253:236-48.

16. Wong FH, Hu CP, Chiu JH, Huang BS, Chang JP, Lin PJ, et al. Expression of multiple oncogenes in human esophageal carcinomas. Cancer Invest. 1994;12: 121-31.

17. Hsu HS, Lin JH, Huang WC, Hsu TW, Chiou SH, Tsai YT, et al. Chemo-resistance of lung cancer stem-like cells depends on activation of Hsp27. Cancer. 2011;117:1516-28

18. Wang X, Proud CG. Methods for studying signal-dependent regulation of translation factor activity. Methods Enzymol. 2007;431:113-42.

19. Kao CL, Hsu HS, Chen HW, Cheng TH. Rapamycin increases the p53/MDM2 protein ratio and p53-dependent apoptosis by translational inhibition of $m d m 2$ in cancer cells. Cancer Lett. 2009;286:250-9.

20. Leu SJJ, Sung JS, Chen MY, Chen CW, Cheng JY, Wang TY, et al. The matricel lular protein CCN1 suppresses lung cancer cell growth by inducing senescence via the p53/p21 pathway. J Cell Biochem. 2013;114:2082-93.

21. Gao N, Flynn DC, Zhang Z, Zhong XS, Walker V, Liu KJ, et al. G1 cell cycle progression and the expression of G1 cyclins are regulated by PI3K/AKT/ mTOR/p70S6K1 signaling in human ovarian cancer cells. Am J Physiol Cell Physiol. 2004;287:C281-91.

22. Sonenberg N, Gingras AC. The mRNA 5' cap-binding protein eIF4E and control of cell growth. Curr Opin Cell Biol. 1998;10:268-75.

23. Rolli-Derkinderen M, Machavoine F, Baraban JM, Grolleau A, Beretta L, Dy M. ERK and p38 inhibit the expression of 4E-BP1 repressor of translation through induction of Egr-1. J Biol Chem. 2003;278:18859-67.

24. Salehi Z, Mashayekhi F. Expression of the eukaryotic translation initiation factor 4E (eIF4E) and 4E-BP1 in esophageal cancer. Clin Biochem. 2006;39:404-9.

25. Grosso S, Pesce E, Brina D, Beugnet A, Loreni F, Biffo S. Sensitivity of global translation to mTOR inhibition in REN cells depends on the equilibrium between eIF4E and 4E-BP1. PLoS One. 2011;6:e29136.

26. Mamane Y, Petroulakis E, Martineau Y, Sato TA, Larsson O, Rajasekhar VK et al. Epigenetic activation of a subset of mRNAs by eIF4E explains its effects on cell proliferation. PLoS One. 2007;2:e242.

27. Seufferlein T, Rozzengurt E. Rapamycin inhibits constitutive $\mathrm{p} 70^{\mathrm{s} 6 \mathrm{k}}$ phosphorylation, cell proliferation, and colony formation in small cell lung cancer cells Cancer Res. 1996;56:3895-7.

28. van der Poel HG, Hanrahan C, Zhong H, Simons JW. Rapamycin induces Smad activity in prostate cancer cell lines. Urol Res. 2003;30:380-6.

29. O'Reilly KE, Warycha M, Davies MA, Rodrik V, Zhou XK, Yee H, et al. Phosphorylated 4E-BP1 is associated with poor survival in melanoma. Clin Cancer Res. 2009; 15:2872-8.

30. She QB, Halilovic E, Ye Q, Zhen W, Shirasawa S, Sasazuki T, et al. 4E-BP1 is a key effector of the oncogenic activation of the AKT and ERK signaling pathways that integrates their function in tumors. Cancer Cell. 2010;18:39-51.

31. Harada T, Morroka T, Ogawa S, Nishida E. ERK induces p35, a neuron-specific activator of cdk5, through induction of egr-1. Nat Cell Biol. 2001;3:453-9.

32. Adamson E, de Belle I, Mittal S, Wang Y, Hayakawa K, Korkmaz K, et al. Egr-1 signaling in prostate cancer. Cancer Biol Ther. 2003;2:617-22.

33. Okamoto I, Doi T, Ohtsu A, Miyazaki M, Tsuya A, Kurei K, et al. Phase I clinical and pharmacokinetic study of RAD001 (Everolimus) administered daily to Japanese patients with advanced solid tumors. Jpn J Clin Oncol. 2010;40:17-23.

34. Werner D, Atmaca A, Pauligk C, Pustowka A, Jager E, Al-Batran SE. Phase I study of everolimus and mitomycin $\mathrm{C}$ for patients with metastatic esophagogastric adenocarcinoma. Cancer Med. 2013;2:325-33. 